\title{
Acute right ventricular dysfunction in severe COVID-19 pneumonia
}

Yun-Long Li ${ }^{1}{ }^{\dagger}$, Jun-Bo Zheng ${ }^{1, \dagger}, \mathrm{Yu}_{\mathrm{Jin}}{ }^{2}$, Rong Tang ${ }^{1}$, Ming Li ${ }^{1}$, Chun-Hong Xiu ${ }^{3}$, Qing-Qing Dai ${ }^{1}$, Shu Zuo ${ }^{1}$, Huai-Quan Wang ${ }^{1}$, Hong-Liang Wang ${ }^{1}$, Ming-Yan Zhao ${ }^{4}$, Ming Ye ${ }^{1, *}$ and Kai-Jiang Yu ${ }^{4, *}$

${ }^{1}$ Department of Intensive Care Medicine, The Second Affiliated Hospital of Harbin Medical University, Harbin, 150081,

Heilongiiang, P. R. China

${ }^{2}$ Department of General Surgery, General Hospital of Heilongjiang Province Land Reclamation Bureau, Harbin, 150088,

Heilongjiang, P. R. China

${ }^{3}$ Department of Echocardiography Lab, The First Affiliated Hospital of Harbin Medical University, Harbin, 150001,

Heilongjiang, P. R. China

${ }^{4}$ Department of Intensive Care Medicine, The First Affiliated Hospital of Harbin Medical University, Harbin, 150001,

Heilongjiang, P. R. China

*Correspondence: yymm1976@sina.com (Ming Ye); drkaijiang1@126.com (Kai-Jiang Yu)

†These authors contributed equally.

\section{DOI: $10.31083 /$ i.rcm.2020.04.159}

This is an open access article under the CC BY 4.0 license (https://creativecommons.org/licenses/by/4.0/).

To investigate the right heart function in coronavirus disease 2019 (COVID-19) patients with acute respiratory distress syndrome (ARDS), a retrospective analysis of 49 COVID-19 patients with ARDS was performed. Patients were divided into severe group and critically-severe group according to the severity of illness. Age-matched healthy volunteers were recruited as a control group. The cardiac cavity diameters, tricuspid annular plane systolic excursion (TAPSE), tricuspid valve regurgitation pressure gradient biggest (TRPG), pulmonary arterial systolic pressure (PASP), maximum inferior vena cava diameter (IVCmax) and minimum diameter (IVCmin), and inferior vena cava collapse index (ICV-CI) were measured using echocardiography. We found that the TAPSE was significantly decreased in pneumonia patients compared to healthy subjects $(P<0.0001)$, and it was significantly lower in critically-severe patients $(P=0.0068)$. The TAPSE was less than $17 \mathrm{~mm}$ in three $(8.6 \%)$ severe and five $(35.7 \%)$ critically-severe patients. In addition, the TAPSE was significantly decreased in severe ARDS patients than in mild ARDS patients. The IVCmax and IVCmin were significantly increased in critically-severe patients compared to healthy subjects and severe patients $(P<0.01)$, whereas the ICV-Cl was significantly decreased $(P<0.05)$. COVID-19 patients had significantly larger right atrium and ventricle than healthy controls $(P<0.01)$. The left ventricular ejection fraction (LVEF) in critically-severe patients was significantly lower than that in severe patients and healthy controls $(P<0.05)$. Right ventricular function was impaired in critically-severe COVID-19 patients. The assessment and protection of the right heart function in COVID-19 patients should be strengthened.

\section{Keywords}

Coronavirus disease 2019; acute respiratory distress syndrome; right ventricular function; echocardiography; retrospective study

\section{Introduction}

Coronavirus disease 2019 (COVID-19) pneumonia, caused by the SARS coronavirus 2 (SARS-CoV-2), has spread rapidly all over the world (Q. Li et al., 2020; WHO, 2020). This disease can induce systemic multiple organ damage, especially in the lungs ( $\mathrm{Wu}$ and McGoogan, 2020). Yang et al. (2020) reported that 26.1\% of patients required admission to an intensive care unit (ICU) and $61.1 \%$ developed acute respiratory distress syndrome (ARDS). Moreover, $81 \%$ of patients who died from COVID-19 developed ARDS, compared to $45 \%$ in those who survived COVID-19 (D. Wang et al., 2020).

Previous research shows that ARDS is often accompanied by right ventricular (RV) dysfunction and the incidence is between 2030\% (Boissier et al., 2013; Mekontso Dessap et al., 2016; Repesse et al., 2016). RV dysfunction is an important determinant of mortality in ARDS patients. However, the incidence of RV dysfunction in patients with ARDS caused by COVID-19 pneumonia is unclear. At present, echocardiography is the preferred choice for evaluating RV function of patients with ARDS, because it is delivered at the bedside, and it is non-invasive and convenient. Tricuspid annular plane systolic excursion (TAPSE) is widely used as a quantitative index to evaluate RV function, which is highly correlated with the mortality of ARDS patients, thus providing a good parameter for to interpret RV function in COVID-19 patients (Wadia et al., 2016). 
Table 1. Clinical characteristics of healthy subjects and patients with COVID-19 pneumonia.

\begin{tabular}{|c|c|c|c|}
\hline & Healthy $(n=25)$ & Severe patients $(\mathrm{n}=35)$ & critically-severe $(n=14)$ \\
\hline Male & $12(48.0 \%)$ & $19(54.3 \%)$ & $6(42.9 \%)$ \\
\hline Age & $62.2 \pm 8.2$ & $64.3 \pm 13.0$ & $65.6 \pm 14.6$ \\
\hline HR & $72.5 \pm 8.9$ & $77.4 \pm 13.6$ & $88.0 \pm 22.6 *$ \\
\hline MAP & $76.5 \pm 6.1$ & $90.7 \pm 12.1 *$ & $78.1 \pm 18.5^{\#}$ \\
\hline APACHE II & - & $6.2 \pm 3.7$ & $15.1 \pm 9.5^{\#}$ \\
\hline Mechanical ventilation & - & - & $11(78.6 \%)$ \\
\hline Invasive ventilation & - & 0 & $14(100 \%)$ \\
\hline Non-invasive ventilation & - & $26(74.3 \%)$ & 0 \\
\hline PEEP $\left(\mathrm{cmH}_{2} \mathrm{O}\right)$ & - & $5.8 \pm 1.6$ & $13.1 \pm 2.6^{\#}$ \\
\hline Vasoactive agent & - & - & $8(57.1 \%)$ \\
\hline $\mathrm{PaO}_{2}: \mathrm{FiO}_{2}$ ratio & - & $189.1 \pm 36.8$ & $92.3 \pm 34.2^{\#}$ \\
\hline \multicolumn{4}{|l|}{ ARDS } \\
\hline Mild & - & $22(62.9 \%)$ & - \\
\hline Moderate & - & $13(37.1 \%)$ & $4(28.6 \%)$ \\
\hline Severe & - & - & $10(71.4 \%)$ \\
\hline \multicolumn{4}{|l|}{ Co-morbidities } \\
\hline Hypertension & - & $12(34.3 \%)$ & $5(35.7 \%)$ \\
\hline Diabetes & - & $8(22.9 \%)$ & $5(35.7 \%)$ \\
\hline Cardiovascular disease & - & $6(17.1 \%)$ & $2(14.3 \%)$ \\
\hline
\end{tabular}

Note: HR: heart rate; MAP: mean arterial pressure; APACHE II: Acute Physiology and Chronic Health Evaluation II; ARDS: acute respiratory distress syndrome. ${ }^{*} P<0.05$ compared with healthy group; ${ }^{\#} P<0.05$ compared with severe patients group.

In this study, we sought to determine the incidence of RV dysfunction using echocardiography in a cohort of patients with ARDS caused by COVID-19 pneumonia.

\section{Methods}

\subsection{Study population}

We retrospectively analyzed 49 patients (age $\geq 18$ years) with ARDS caused by COVID-19 pneumonia who were admitted to the ICU of the First Affiliated Hospital of Harbin Medical University from February 2020 to March 2020. The study was approved by the ethics committee of the Second Affiliated Hospital of Harbin Medical University (KY2020-015). The results of novel coronavirus nucleic acid test were positive in all patients. COVID-19 patients with ARDS were enrolled in our study. The diagnosis of ARDS was based on the Berlin definition (Force et al., 2012). According to the guidelines for diagnosis and treatment of novel coronavirus pneumonia (Trial Version 5) (National health commission of the People's Republic of China, 2020), all enrolled patients were divided into severe and critically-severe groups. The guideline was released by National Health Commission \& State Administration of Traditional Chinese Medicine, and is now widely applied in published studies (C. Wang et al., 2020; Wu and McGoogan, 2020). Patients with polypnea (respiratory rate $\geq 30$ times $/ \mathrm{min}$ ), decreased oxygen saturation ( $\leq 93 \%$ at rest), $\mathrm{PaO}_{2} / \mathrm{FiO}_{2} \leq 300 \mathrm{mmHg}$ or exacerbation of lung imaging more than $50 \%$ within $24-48$ hours were classified as the severe type, and those with respiratory failure requiring mechanical ventilation, shock or other organ failure were classified as the critically-severe type. Patients with no echocardiographic windows, or who had congenital heart disease, cardiac valvular disease, pulmonary thrombosis or acute myocardial infarction were excluded. All 49 patients were enrolled in the final analy- sis, including 35 severe and 14 critically-severe cases. Age, gender, underlying disease, APACHE II score within 24 hours of hospitalization, heart rate (HR) and mean arterial pressure (MAP) during echocardiographic examination were recorded. We also analyzed all the data of patients according to the severity of ARDS. Age-matched healthy volunteers $(n=25)$ were recruited as the control group.

\subsection{Echocardiography}

Echocardiography assessment was performed in all patients within $48 \mathrm{~h}$ of ICU admission by the same echocardiographer using a commercially available system (Vivid E9, GE, Inc.) equipped with a 2.5-4 MHz transducer (M5S probe). All patients were examined in a calm or sedated state. All echocardiographic parameters were evaluated according to the guidelines of American Society of Echocardiography (ASE) (Lang et al., 2015).

\section{$2.3 R V$ function assessment}

TAPSE was defined as the peak excursion of tricuspid annulus from end-diastole to end-systole and was measured from the apical four-chamber view by placing a M-mode cursor through the lateral tricuspid annulus. The TAPSE less than $17 \mathrm{~mm}$ indicates RV dysfunction (Lang et al., 2015).

The diameter changes of inferior vena cava (IVC) during the respiratory cycle were measured from the IVC long axis view by Mmode. When breathing spontaneously, the minimum diameter of the inferior vena cava (IVCmin) was measured in the inspiratory phase and the maximum diameter of the inferior vena cava (IVCmax) was measured in the expiratory phase. The inferior vena cava collapse index (ICV-CI) was calculated by the formula of (IVCmax-IVCmin) $/$ IVCmax $\times 100 \%$.

The diameter of pulmonary artery (PAD) was measured from parasternal short-axis view by two-dimensional echocardiography. 

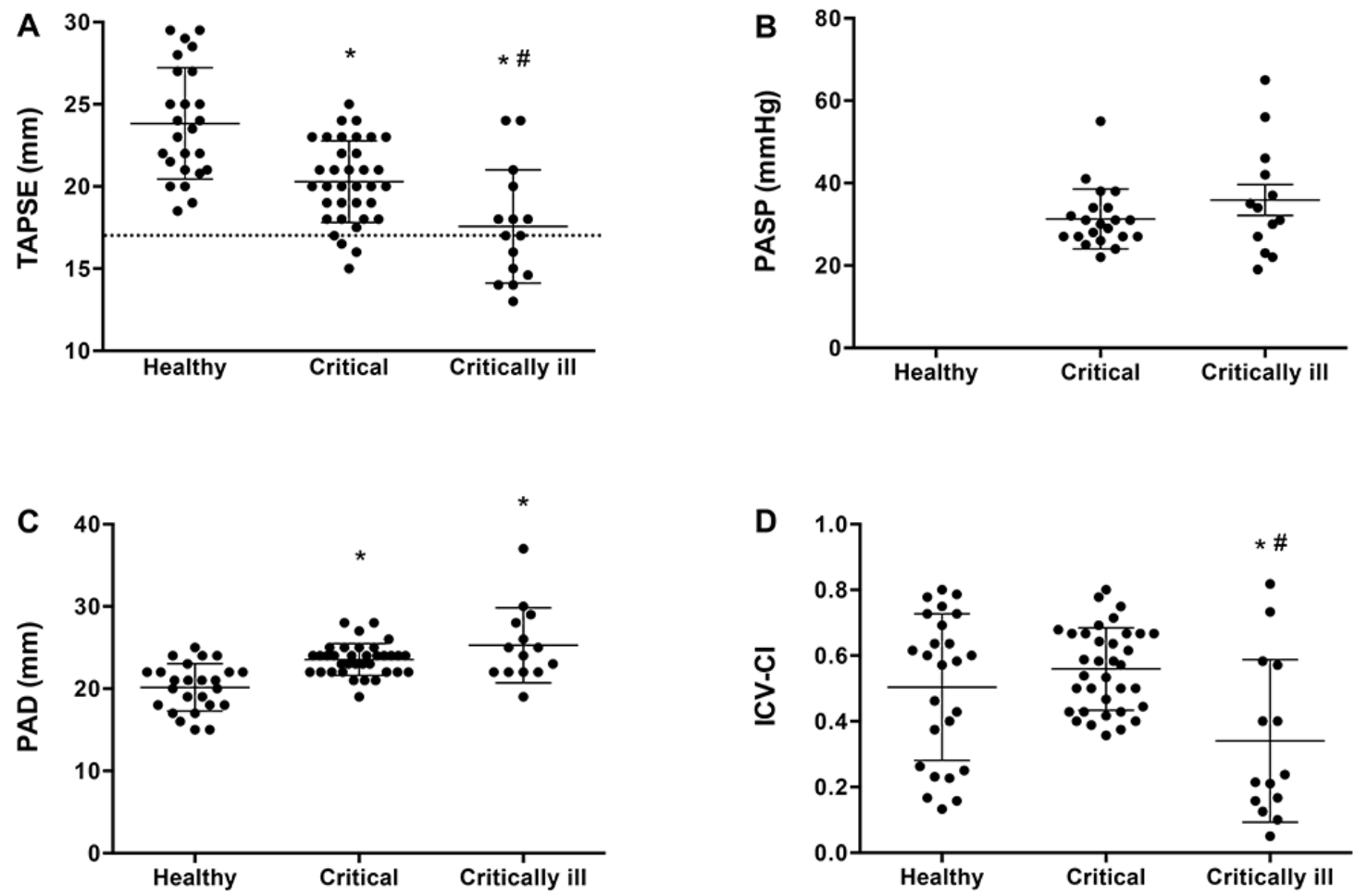

Fig. 1. Values of TAPSE (A), PASP (B), PAD (C) and ICV-CI (D) for individual patients with COVID-19 pneumonia and healthy subjects. The dotted line in (A) indicates $17 \mathrm{~mm}$ of TAPSE. TAPSE: tricuspid annular plane systolic excursion; PASP: pulmonary arterial systolic pressure; PAD: pulmonary artery diameter; ICV-CI: inferior vena cava collapse index. ${ }^{*} P<0.05$ compared with healthy group; ${ }^{\#} P<0.05$ compared with severe patients group.

Tricuspid valve regurgitation pressure gradient biggest (TRPG) was measured and pulmonary arterial systolic pressure (PASP) was estimated indirectly by tricuspid regurgitation velocity with the addition of right atrial pressure, assuming no significant right ventricular outflow tract (RVOT) obstruction.

\subsection{Cardiac diameters and left ventricular function}

By two-dimensional echocardiography, left ventricular enddiastolic dimension (LVEDD) was measured from the parasternal long-axis view, while the right atrial diameter (RAD) and right ventricular diameter (RVD) were measured from the apical fourchamber view. The sampling volume was placed above the aortic valve orifice to obtain the aortic valve blood flow spectrum and measure the aortic valve velocity (AV) from the apical five-chamber view. A modified Simpson's method was used to measure left ventricular end-diastolic volume (EDV) and end-systolic volume (ESV). Left ventricular ejection fraction (LVEF) was calculated as followed: $\mathrm{LVEF}=(\mathrm{EDV}-\mathrm{ESV}) / \mathrm{EDV}$.

\subsection{Statistical analysis}

The statistical analysis was performed using GraphPad Prism 6. Categorical variables are reported as frequencies and percentages. Continuous variables were reported as mean \pm standard deviation (SD). Categorical variables were compared using Chi square test. For comparison between severe and critically-severe patients, the Mann-Whitney U test was used. One-way ANOVA followed by Tukey's test was used for statistical comparisons between healthy subjects, and the severe and critically-severe groups.

\section{Results \\ 3.1 Patient characteristics}

Among all patients enrolled, 35 (71.4\%) were classified under the severe type of COVID-19 pneumonia and 14 (28.6\%) were classified under the critically-severe type. There were 22 (62.9\%) patients with mild and $13(37.1 \%)$ with moderate ARDS in the severe patients, four $(28.6 \%)$ with moderate, and $10(71.4 \%)$ with severe ARDS in critically-severe patients. The clinical characteristics of patients are shown in (Table 1).

There was no significant difference in age and biological sex between COVID-19 pneumonia patients and healthy donors $(P>$ 0.05). Critically-severe patients had significantly higher APACHE II score $(P=0.0001)$ and lower $\mathrm{PaO}_{2} / \mathrm{FiO}_{2}$ compared to severe patients $(P<0.0001)$. The HR in critically-severe patients was significantly increased compared to the healthy group $(P=0.0056)$, and MAP was significantly decreased than that in the healthy group and criticallysevere group $(P<0.01)$. In critically-severe patients, $11(78.6 \%)$ cases received invasive mechanical ventilation and eight $(57.1 \%)$ were treated with vasoactive drugs. There was no statistically significant difference in comorbidity between severe and critically-severe patients $(P>0.05)$.

\subsection{RV function}

Values of TAPSE for individual subjects are shown in (Fig. 1A). A normal TAPSE was recorded in all healthy subjects, and the TAPSE was significantly decreased in patients with COVID19 pneumonia. Further, the TAPSE was significantly lower in critically-severe patients than in severe patients $(P=0.0128)$. Three $(8.6 \%)$ severe and five $(35.7 \%)$ critically-severe patients had a re- 
Table 2. Echocardiographic parameters of right ventricular function.

\begin{tabular}{lccc}
\hline & Healthy $(\mathrm{n}=25)$ & Severe patients $(\mathrm{n}=35)$ & critically-severe $(\mathrm{n}=14)$ \\
\hline PAD $(\mathrm{mm})$ & $20.2 \pm 2.9$ & $23.7 \pm 2.0 *$ & $25.3 \pm 4.6 *$ \\
TRPG $(\mathrm{mmHg})$ & - & $25.3 \pm 5.2$ & $28.8 \pm 11.0$ \\
PASP $(\mathrm{mmHg})$ & - & $29.8 \pm 4.8$ & $35.9 \pm 13.5$ \\
TAPSE $(\mathrm{mm})$ & $23.8 \pm 3.4$ & $20.4 \pm 2.4^{*}$ & $17.6 \pm 3.4 * \#$ \\
IVCmax (mm) & $14.4 \pm 3.5$ & $14.0 \pm 2.0$ & $17.6 \pm 3.8 * \#$ \\
IVCmin (mm) & $7.7 \pm 4.8$ & $6.2 \pm 2.1$ & $12.1 \pm 5.7 * \#$ \\
ICV-CI $(\%)$ & $50.4 \pm 22.3$ & $55.9 \pm 12.6$ & $34.1 \pm 24.8 * \#$ \\
\hline
\end{tabular}

Note: PAD: pulmonary artery diameter; TRPG: tricuspid valve regurgitation pressure gradientbiggest; PASP: pulmonary arterial systolic pressure; TAPSE: tricuspid annular plane systolic excursion; IVCmax: maximum diameter of the inferior vena cava; IVCmin: minimum diameter of the inferior vena cava; ICV-CI: inferior vena cava collapse index. ${ }^{*} P<0.05$ compared with healthy group; ${ }^{\#} P<0.05$ compared with severe patients group.

Table 3. Cardiac diameters and left ventricular function.

\begin{tabular}{lccc}
\hline & Healthy $(\mathrm{n}=25)$ & Severe patients $(\mathrm{n}=35)$ & critically-severe $(\mathrm{n}=14)$ \\
\hline LVEDD $(\mathrm{mm})$ & $43.6 \pm 3.5$ & $45.6 \pm 2.9$ & $44.3 \pm 3.0$ \\
RVD $(\mathrm{mm})$ & $17.6 \pm 3.1$ & $34.7 \pm 3.3 *$ & $34.6 \pm 3.3 *$ \\
RAD $(\mathrm{mm})$ & $30.4 \pm 3.4$ & $32.7 \pm 2.3 *$ & $34.1 \pm 3.6 *$ \\
LVEF $(\%)$ & $64.8 \pm 4.0$ & $63.9 \pm 5.0$ & $59.4 \pm 8.4 * \#$ \\
AV $(\mathrm{cm} / \mathrm{s})$ & $117.4 \pm 9.3$ & $112.7 \pm 16.1$ & $112.1 \pm 16.3$ \\
\hline
\end{tabular}

Note: LVEDD: left ventricular end-diastolic dimension; RVD: right ventricular diameter; RAD: right atrial diameter; LVEF: left ventricular ejection fraction; $\mathrm{AV}$ : aortic valve velocity. ${ }^{*} P<$ 0.05 compared with healthy group; $\# P<0.05$ compared with severe patients group.

duced TAPSE $(<17 \mathrm{~mm})$.

The echocardiographic parameters of RV function in patients with COVID-19 pneumonia are shown in (Table 2) and (Fig. 1BD). Tricuspid regurgitation was present in $21(60.0 \%)$ severe and $13(92.9 \%)$ critically-severe patients, and there were no statistically significant differences in PASP and TRPG between the two groups $(P>0.05)$. Patients with COVID-19 pneumonia had a significantly higher PAD than healthy subjects $(P<0.0001)$. The IVCmax and IVCmin were significantly increased in critically-severe patients than in healthy subjects and severe patients $(P<0.01)$, whereas ICV-CI was significantly decreased $(P<0.05)$.

\subsection{The diameters of cardiac cavity and LVEF}

The diameters of cardiac cavity and other echocardiographic parameters are shown in (Table 3). Patients with COVID-19 pneumonia had significantly larger right atrium and right ventricle than healthy subjects $(P<0.01)$, and there was no statistically significant difference in RAD and RVD between severe and critically-severe patients $(P>0.05)$. Critically-severe patients had a significantly lower LVEF than healthy subjects and severe patients $(P<0.05)$.

\subsection{Parameters in COVID-19 pneumonia patients being classified by ARDS severity}

There were 22 patients with mild, 17 with moderate and 10 with severe ARDS in the selected COVID-19 pneumonia population. The clinical characteristics and echocardiographic parameters in COVID-19 pneumonia patients being classified by ARDS severity are shown in (Table 4).

Severe ARDS patients had significantly higher APACHE II score and lower $\mathrm{PaO}_{2} / \mathrm{FiO}_{2}$ compared to mild and moderate ARDS patients, and moderate ARDS patients had lower $\mathrm{PaO}_{2} / \mathrm{FiO}_{2}$ compared to mild ARDS patients $(P<0.0001)$. Thirteen $(59.1 \%)$ mild and $13(76.5 \%)$ moderate ARDS patients received non-invasive ventilation, four (23.5\%) moderate and 10 (100\%) severe ARDS patients received invasive ventilation. Significant higher PEEP was applied in severe ARDS patients. The TAPSE was significantly decreased in severe ARDS patients than mild ARDS patients. All patients showed a significant larger PAD, RAD and RVD than healthy subjects regardless of the severity of ARDS.

\section{Discussion}

Our results demonstrated that RV dysfunction is an important clinical feature in COVID-19 pneumonia patients with ARDS, which is related to the severity of COVID-19 pneumonia and ARDS. Bedside echocardiography allows for an early integrated assessment of the RV function through the evaluation of TAPSE. The echocardiographic parameters of RV function and cardiac cavity in COVID19 pneumonia patients show abnormity to different extents compared to healthy subjects.

Viral pneumonia is the one of the most common causes for ARDS and is associated with high morbidity and mortality worldwide, such as in SARS, and H1N1 (Brown et al., 2011; S. Li et al., 2020; Viasus et al., 2011). RV dysfunction is frequently presented in these patients, and contributes to left-right asymmetry of the heart, which can worsen hemodynamic stability and is associated with a poorer prognosis (Arrigo et al., 2019; Biswas, 2015; Repesse et al., 2016). In our study, a higher incidence of RV dysfunction was found 
Table 4. Clinical characteristics and echocardiographic parameters in COVID-19 pneumonia patients being classified by ARDS severity.

\begin{tabular}{|c|c|c|c|c|}
\hline & Healthy $(\mathrm{n}=25)$ & Mild $(\mathrm{n}=22)$ & Moderate $(n=17)$ & Severe $(n=10)$ \\
\hline Male & $12(48.0 \%)$ & $12(54.5 \%)$ & $9(52.9 \%)$ & $4(40.0 \%)$ \\
\hline Age & $62.2 \pm 8.2$ & $67.1 \pm 13.9$ & $60.2 \pm 9.5$ & $66.8 \pm 16.9$ \\
\hline HR & $72.5 \pm 8.9$ & $79.0 \pm 14.3$ & $74.8 \pm 12.0$ & $93.00 \pm 24.3 * \dagger$ \\
\hline MAP & $76.5 \pm 6.1$ & $93.6 \pm 12.7 *$ & $84.6 \pm 10.1$ & $77.0 \pm 20.9^{\#}$ \\
\hline APACHE II & - & $6.2 \pm 4.0$ & $6.9 \pm 3.3$ & $17.4 \pm 10.4 \# \dagger$ \\
\hline \multicolumn{5}{|l|}{ Mechanical ventilation } \\
\hline Invasive ventilation & - & 0 & $4(23.5 \%)$ & $10(100 \%)$ \\
\hline Non-invasive ventilation & - & $13(59.1 \%)$ & $13(76.5 \%)$ & 0 \\
\hline $\operatorname{PEEP}\left(\mathrm{cmH}_{2} \mathrm{O}\right)$ & & $4.8 \pm 1.1$ & 7. $5 \pm 1.9^{\#}$ & $14.4 \pm 2.0 \# \dagger$ \\
\hline $\mathrm{PaO}_{2}: \mathrm{FiO}_{2}$ ratio & - & $214.7 \pm 10.0$ & $143.1 \pm 24.5^{\#}$ & $75.7 \pm 13.7 \mathrm{\# \dagger}$ \\
\hline \multicolumn{5}{|l|}{ Co-morbidities } \\
\hline Hypertension & - & $7(31.8 \%)$ & $6(35.3 \%)$ & $4(40.0 \%)$ \\
\hline Diabetes & - & $4(18.2 \%)$ & $4(23.5 \%)$ & $5(50.0 \%)$ \\
\hline Cardiovascular disease & - & $4(18.2 \%)$ & $3(17.6 \%)$ & $1(10.0 \%)$ \\
\hline \multicolumn{5}{|l|}{ Echocardiographic data } \\
\hline TAPSE $(\mathrm{mm})$ & $23.8 \pm 3.4$ & $20.2 \pm 2.4 *$ & $19.8 \pm 3.2 *$ & $16.8 \pm 2.6 * \#$ \\
\hline PAD (mm) & $20.2 \pm 2.9$ & $23.8 \pm 2.2 *$ & $23.6 \pm 1.9 *$ & $25.4 \pm 5.2 *$ \\
\hline TRPG (mmHg) & - & $25.5 \pm 5.4$ & $27.2 \pm 10.3$ & $30.8 \pm 11.0$ \\
\hline PASP (mmHg) & - & $29.6 \pm 5.3$ & $32.5 \pm 10.8$ & $36.6 \pm 13.3$ \\
\hline IVCmax (mm) & $14.4 \pm 3.5$ & $13.9 \pm 2.0$ & $15.2 \pm 2.6$ & $17.3 \pm 4.4 \#$ \\
\hline IVCmin (mm) & $7.7 \pm 4.8$ & $6.0 \pm 2.1$ & $8.7 \pm 4.6$ & $10.6 \pm 6.0 \#$ \\
\hline $\operatorname{ICV}-\mathrm{CI}(\%)$ & $50.4 \pm 22.3$ & $57.1 \pm 11.8$ & $44.4 \pm 21.6$ & $42.3 \pm 24.7$ \\
\hline LVEDD (mm) & $43.6 \pm 3.5$ & $45.2 \pm 3.1$ & $45.4 \pm 3.0$ & $44.9 \pm 3.0$ \\
\hline $\mathrm{RVD}(\mathrm{mm})$ & $17.6 \pm 3.1$ & $34.5 \pm 3.8 *$ & $34.4 \pm 3.2 *$ & $34.7 \pm 3.0 *$ \\
\hline RAD (mm) & $30.4 \pm 3.4$ & $32.6 \pm 2.1 *$ & $32.8 \pm 2.5 *$ & $34.7 \pm 3.9 *$ \\
\hline $\operatorname{LVEF}(\%)$ & $64.8 \pm 4.0$ & $64.9 \pm 5.1$ & $61.5 \pm 4.7$ & $59.6 \pm 9.8$ \\
\hline $\mathrm{AV}(\mathrm{cm} / \mathrm{s})$ & $117.4 \pm 9.3$ & $113.3 \pm 16.7$ & $110.4 \pm 17.4$ & $114.7 \pm 13.0$ \\
\hline
\end{tabular}

Note: ARDS: acute respiratory distress syndrome; HR: heart rate; MAP: mean arterial pressure; APACHE II: Acute Physiology and Chronic Health Evaluation II; PAD: pulmonary artery diameter; TRPG: tricuspid valve regurgitation pressure gradientbiggest; PASP: pulmonary arterial systolic pressure; TAPSE: tricuspid annular plane systolic excursion; IVCmax: maximum diameter of the inferior vena cava; IVCmin: minimum diameter of the inferior vena cava; ICV-CI: inferior vena cava collapse index; LVEDD: left ventricular end-diastolic dimension; RVD: right ventricular diameter; RAD: right atrial diameter; LVEF: left ventricular ejection fraction; AV: aortic valve velocity. ${ }^{*} P<0.05$ compared with healthy group; ${ }^{\#} P<0.05$ compared with mild ARDS patients; ${ }^{\dagger} P<0.05$ compared with moderate ARDS patients.

in critically-severe group of COVID-19 pneumonia who had more severe ARDS, which is a finding similar to that in the severe ARDS population caused by SARS and H1N1 viruses (Shorr et al., 2017; Viasus et al., 2011; Yin and Wunderink, 2018). Thus, RV dysfunction is common in viral pneumonia patients, especially in patients with severe ARDS.

TAPSE is the most commonly used index for evaluating RV function. This index has been used in patients with ARDS and other clinical conditions, e.g. in patients with cardiac surgery (Wranne et al., 1991), pulmonary embolism (Deng and Peng, 2010), and RV myocardial infarction (Alam et al., 2000). A TAPSE less than 17 $\mathrm{mm}$ was recommended as a major parameter of RV dysfunction by ASE guidelines. In present study, we found the TAPSE in patients with COVID-19 pneumonia was significantly decreased compared to healthy people, and the proportion of patients with TAPSE < $17 \mathrm{~mm}$ was significantly higher in critically-severe patients (35.7\%) than that in severe patients $(8.6 \%)$, indicating that there was a correlation between the occurrence of RV dysfunction and the severity of
COVID-19 pneumonia. Moreover, severe ARDS patients caused by COVID-19 pneumonia had the worst TAPSE, which may because of significantly higher PEEP and lower level of oxygenation in these patients. Severe hypoxemia and high PEEP ventilation have been shown to induce pulmonary vasoconstriction (Beitler et al., 2016; Price et al., 2010), and cause RV dysfunction. RV function was indicated by TAPSE in several previous studies (Lazzeri et al., 2018; Szekely et al., 2020; Zochios et al., 2017). The assessment of the TAPSE by echocardiography in COVID-19 disease is important in the management of deteriorating patients, allowing early identification and treatment of cardiac injury. Patients in the critically-severe group appeared to be more severely ill in terms of APACHE II, and had a lower MAP and higher HR. Moreover, invasive ventilation and vasopressors are frequently required in these patients.

The function of the RV is to keep proper pulmonary perfusion pressure and systemic venous pressure to maintain normal blood flow (Zochios and Jones, 2014). The main factors affecting the RV function in patients with ARDS include impaired RV contrac- 
tion and increased pulmonary vascular resistance. Previous research has indicated that an increase of pulmonary vascular resistance may be induced by higher PEEP, vasoconstrictor, hypoxemia, hypercapnia, and acidosis, which can deteriorate right heart structure and function (Price et al., 2012, 2010). In our study, we found that decreased TAPSE was associated with higher PEEP. Recent studies have shown that the clinical characteristics of severe and criticallysevere COVID-19 pneumonia patients include severe hypoxemia, hypercapnia and microthrombosis, which may lead to higher pulmonary vascular resistance (Lazzeri et al., 2020; D. Wang et al., 2020; Wu and McGoogan, 2020; Yang et al., 2020).

In our study, the values of TRPG and PASP were higher than normal ranges and increased in COVID-19 pneumonia patients. Although the majority of patients in the critically-severe group received mechanical ventilation, there were no significant differences in TRPG and PASP between severe and critically-severe patients. This finding might be explained by the fact that pulmonary vascular resistance does not significantly increase by positive pressure mechanical ventilation and is not seriously deteriorated in the early stages of the disease (Paternot et al., 2016). The right heart had a poor ability to regulate pressure load (Mekontso Dessap et al., 2016). In this study, the right heart cavity and pulmonary artery were enlarged soon after the increase of pulmonary vascular resistance in patients with COVID-19 pneumonia.

Patients with critically-severe COVID-19 pneumonia are often accompanied by organ dysfunction and shock. The results of the present study have shown that both RV and left ventricular systolic function in critically-severe patients with COVID-19 pneumonia are impaired. The hemodynamic instability of decreased LVEF and MAP is associated with more severe RV dysfunction, which present significantly decreased TAPSE, enhanced diameters of IVC and reduced ICV-CI. Therefore, the monitoring and protection of right ventricular function need to be strengthened in the management of patients with COVID-19 pneumonia.

There are limitations in the present study. This is a retrospective study with limited sample size. Unfortunately, the representative TAPSE ultrasound images were not saved and some parameters were not routinely measured in all selected patients, such as RV fraction area change, cardiac output, left ventricular fraction shortening. The results need to be further confirmed by prospective clinical studies. ARDS often causes right heart dysfunction rather than left heart dysfunction. A recent study suggested that left ventricular systolic function is preserved in the majority of patients with COVID-19 infection, but RV function is impaired (Szekely et al., 2020). Thus, we paid more attention to the echocardiographic data of right heart. It is unknown whether RV dysfunction is worst in ARDS caused by the COVID-19 infection compared with other etiologies. Future studies may be required to investigate RV function in different medical conditions. In addition, although echocardiography was performed by ultrasonologist, it became more challenging than usual to obtain parameters since three-level isolation and protection measures must be taken when contacting patients with COVID-19 pneumonia. Although Speckle tracking echocardiography was reported as a novel echocardiographic technique to assess RV function, it is not routinely used in ARDS patients, especially for critically-ill patients (Bonizzoli et al., 2018). In this case, transthoracic echocardiography is the most important bedside examination method for evaluating pa- tients with COVID-19 pneumonia.

\section{Conclusions}

$\mathrm{RV}$ dysfunction is relatively common in patients with severe and critically-severe COVID-19 pneumonia, which is related to the severity of illness. Early monitoring of RV function is very important for these patients. It is suggested that bedside echocardiography might be used as an objective test in the assessment of RV function in COVID-19 pneumonia patients with ARDS. TAPSE may serve as a quantitative indicator of early detection of RV dysfunction. These findings are helpful to further improve the clinical diagnosis and treatment strategies for patients with COVID-19 pneumonia.

\section{Abbreviations}

ASE: American society of echocardiography; ARDS: Acute respiratory distress syndrome; AV: Aortic valve velocity; COVID-19: Coronavirus disease 2019; EDV: End-diastolic volume; ESV: Endsystolic volume; HR: Heart rate; ICU: Intensive care unit; ICV-CI: Inferior vena cava collapse index; IVC: Inferior vena cava; IVCmax: Maximum diameter of the inferior vena cava; IVCmin: Minimum diameter of the inferior vena cava; LVEDD: Left ventricular enddiastolic dimension; LVEF: Left ventricular ejection fraction; MAP: Mean arterial pressure; PAD: Diameter of pulmonary artery; PASP: Pulmonary arterial systolic pressure; RAD: Right atrial diameter; RV: Right ventricular; RVD: Right ventricular diameter; RVOT: Right ventricular outflow tract; SARS-CoV-2: SARS coronavirus 2; SD: Standard deviation; TAPSE: Tricuspid annular plane systolic excursion; TRPG: Tricuspid valve regurgitation pressure gradient biggest.

\section{Authors' contributions}

Yunlong Li, Ming Li, Rong Tang collected the epidemiological and clinical data. Ming Ye, Hongliang Wang guided the study design. Yunlong Li, Junbo Zheng, Yu Jin, and Chunhong Xiu summarized all data. Yunlong Li, Junbo Zheng and Chunhong Xiu drafted the manuscript. Kaijing Yu, Ming Ye and Mingyan Zhao revised the final manuscript. All authors read and approved the final manuscript.

\section{Ethics approval and consent to participate}

The study was approved by the ethics committee of the Second Affiliated Hospital of Harbin Medical University (KY2020-015).

\section{Acknowledgements}

We thank all patients and healthy volunteers involved in the study.

\section{Conflict of Interest}

The authors declare no conflict of interest.

Submitted: August 03, 2020

Revised: October 28, 2020

Accepted: October 31, 2020

Published: December 30, 2020

\section{References}

Alam, M., Wardell, J., Andersson, E., Samad, B. A. and Nordlander, R. (2000) Right ventricular function in patients with first inferior myocar- 
dial infarction: assessment by tricuspid annular motion and tricuspid annular velocity. American Heart Journal 139, 710-715.

Arrigo, M., Huber, L. C., Winnik, S., Mikulicic, F., Guidetti, F., Frank, M., Flammer, A. J. and Ruschitzka, F. (2019) Right ventricular failure: pathophysiology, diagnosis and treatment. Cardiac Failure Review $\mathbf{5}$, 140-146

Beitler, J. R., Malhotra, A. and Thompson, B. T. (2016) Ventilator-induced lung injury. 37, 633-646.

Biswas, A. (2015) Right heart failure in acute respiratory distress syndrome: an unappreciated albeit a potential target for intervention in the management of the disease. Indian Journal of Critical Care Medicine 19, 606609.

Boissier, F., Katsahian, S., Razazi, K., Thille, A. W., Roche-Campo, F., Leon, R., Vivier, E., Brochard, L., Vieillard-Baron, A., Brun-Buisson, C. and Mekontso Dessap, A. (2013) Prevalence and prognosis of cor pulmonale during protective ventilation for acute respiratory distress syndrome. Intensive Care Medicine 39, 1725-1733.

Bonizzoli, M., Cipani, S., Lazzeri, C., Chiostri, M., Ballo, P., Sarti, A. and Peris, A. (2018) Speckle tracking echocardiography and right ventricle dysfunction in acute respiratory distress syndrome a pilot study. Echocardiography 35, 1982-1987.

Brown, S. M., Pittman, J., Miller Iii, R. R., Horton, K. D., Markewitz, B., Hirshberg, E., Jones, J. and Grissom, C. K. (2011) Right and left heart failure in severe H1N1 influenza a infection. The European Respiratory Journal 37, 112-118.

Deng, S. Q. and Peng, H. J. (2010) Characteristics of and public health responses to the coronavirus disease 2019 outbreak in China. Journal of Clinical Medicine 9, 575

Force, A. D. T., Ranieri, V. M., Rubenfeld, G. D., Thompson, B. T., Ferguson, N. D., Caldwell, E., Fan, E., Camporota, L. and Slutsky, A. S. (2012) Acute respiratory distress syndrome: the Berlin definition. JAMA 307, 2526-2533

Huang, S. J., Nalos, M., Smith, L., Rajamani, A. and McLean, A. S (2018) The use of echocardiographic indices in defining and assessing right ventricular systolic function in critical care research. Intensive Care Medicine 44, 868-883.

Lang, R. M., Badano, L. P., Mor-Avi, V., Afilalo, J., Armstrong, A., Ernande, L., Flachskampf, F. A., Foster, E., Goldstein, S. A., Kuznetsova T., Lancellotti, P., Muraru, D., Picard, M. H., Rietzschel, E. R., Rudski, L., Spencer, K. T., Tsang, W. and Voigt, J. U. (2015) Recommendations for cardiac chamber quantification by echocardiography in adults: an update from the american society of echocardiography and the European association of cardiovascular imaging. European Heart Journal Cardiovascular Imaging 16, 233-270.

Lazzeri, C., Bonizzoli, M., Cianchi, G., Batacchi, S., Chiostri, M. and Peris, A. (2020) Severity of acute respiratory distress syndrome and echocardiographic findings in clinical practice-an echocardiographic pilot study. Heart \& Lung: The Journal of Critical Care 49, 622-625.

Lazzeri, C., Bonizzoli, M., Cianchi, G., Batacchi, S., Terenzi, P., Cozzolino, M., Bernardo, P. and Peris, A. (2018) Lactate and echocardiography before veno-venous extracorporeal membrane oxygenation support. Heart, Lung and Circulation 27, 99-103.

Li, Q., Guan, X., Wu, P., Wang, X., Zhou, L., Tong, Y., Ren, R., Leung, K. S. M., Lau, E. H. Y., Wong, J. Y., Xing, X., Xiang, N., Wu, Y., Li, C., Chen, Q., Li, D., Liu, T., Zhao, J., Liu, M., Tu, W., Chen, C., Jin, L., Yang, R., Wang, Q., Zhou, S., Wang, R., Liu, H., Luo, Y., Liu, Y., Shao, G., Li, H., Tao, Z., Yang, Y., Deng, Z., Liu, B., Ma, Z., Zhang, Y., Shi, G., Lam, T. T. Y., Wu, J. T., Gao, G. F., Cowling, B. J., Yang, B., Leung, G. M. and Feng, Z. (2020) Early transmission dynamics in Wuhan, China, of novel coronavirus-infected pneumonia. The New England Journal of Medicine 382, 1199-1207.

Li, S. H., Hsieh, M. J., Lin, S. W., Chuang, L. P., Lee, C. S., Chiu, L. C., Chang, C. H., Hu, H. C., Huang, C. C. and Kao, K. C. (2020) Outcomes of severe $\mathrm{H} 1 \mathrm{~N} 1$ pneumoniae: a retrospective study at intensive care units. Journal of the Formosan Medical Association = Taiwan Yi Zhi 119, 2633.

Mekontso Dessap, A., Boissier, F., Charron, C., Begot, E., Repesse, X., Legras, A., Brun-Buisson, C., Vignon, P. and Vieillard-Baron, A. (2016)
Acute cor pulmonale during protective ventilation for acute respiratory distress syndrome: prevalence, predictors, and clinical impact. Intensive Care Medicine 42, 862-870.

National health commission of the People's Republic of China. (2020) Guidelines for Diagnosis and Treatment of Novel Coronavirus Pneumonia (Trial Version 5) 2020. http://www.nhc.gov.cn/yzygj/s7653p /202002/d4b895337e19445f8d728fcaf1e3e13a.shtml. (In Chinese)

Paternot, A., Repesse, X. and Vieillard-Baron, A. (2016) Rationale and description of right ventricle-protective ventilation in ARDS. Respiratory Care 61, 1391-1396.

Price, L. C., McAuley, D. F., Marino, P. S., Finney, S. J., Griffiths, M. J. and Wort, S. J. (2012) Pathophysiology of pulmonary hypertension in acute lung injury. American Journal of Physiology Lung Cellular and Molecular Physiology 302, L803-815.

Price, L. C., Wort, S. J., Finney, S. J., Marino, P. S. and Brett, S. J. (2010) Pulmonary vascular and right ventricular dysfunction in adult critical care: current and emerging options for management: a systematic literature review. Critical Care 14, R169.

Repesse, X., Charron, C. and Vieillard-Baron, A. (2016) Acute respiratory distress syndrome: the heart side of the moon. Current Opinion in Critical Care 22, 38-44.

Shorr, A. F., Zilberberg, M. D., Micek, S. T. and Kollef, M. H. (2017) Viruses are prevalent in non-ventilated hospital-acquired pneumonia Respiratory Medicine 122, 76-80.

Szekely, Y., Lichter, Y., Taieb, P., Banai, A., Hochstadt, A., Merdler, I., Gal Oz, A., Rothschild, E., Baruch, G., Peri, Y., Arbel, Y. and Topilsky, Y. (2020) Spectrum of cardiac manifestations in COVID-19: a systematic echocardiographic study. Circulation 142, 342-353.

Viasus, D., Pano-Pardo, J. R., Pachon, J., Campins, A., Lopez-Medrano, F., Villoslada, A., Farinas, M. C., Moreno, A., Rodriguez-Bano, J., Oteo, J. A., Martinez-Montauti, J., Torre-Cisneros, J., Segura, F., Gudiol, F., Carratalà, J., Novel Influenza A (H1N1) Study Group of the Spanish Network for Research in Infectious Diseases (REIPI). (2011) Factors associated with severe disease in hospitalized adults with pandemic (H1N1) 2009 in Spain. Clinical Microbiology and Infection 17, 738-746.

Wadia, S. K., Shah, T. G., Hedstrom, G., Kovach, J. A. and Tandon, R. (2016) Early detection of right ventricular dysfunction using transthoracic echocardiography in ARDS: a more objective approach. Echocardiography 33, 1874-1879.

Wang, C., Fei, D., Li, X., Zhao, M. and Yu, K. (2020) Il-6 May be a good biomarker for earlier detection of COVID-19 progression. Intensive Care Medicine 46, 1475-1476

Wang, D., Hu, B., Hu, C., Zhu, F., Liu, X., Zhang, J., Wang, B., Xiang, H., Cheng, Z., Xiong, Y., Zhao, Y., Li, Y., Wang, X. and Peng, Z. (2020) Clinical characteristics of 138 hospitalized patients with 2019 novel coronavirus-infected pneumonia in Wuhan, China. JAMA $\mathbf{3 2 3}$ 1061-1069.

WHO (2020) World Health Organization. Coronavirus Disease (COVID-2019) Situation Reports. Available at: https: //www.who.int/Emergencies/Diseases/Novel-Coronavirus -2019/Situation-Reports/.

Wranne, B., Pinto, F. J., Hammarström, E., St Goar, F. G., Puryear, J., and Popp, R. L. (1991) Abnormal right heart filling after cardiac surgery: time course and mechanisms. British Heart Journal 66, 435-442.

Wu, Z. and McGoogan, J. M. (2020) Characteristics of and important lessons from the coronavirus disease 2019 (COVID-19) Outbreak in China: summary of a report of 72314 cases from the Chinese center for disease control and prevention. JAMA 323, 1239-1242.

Yang, X., Yu, Y., Xu, J., Shu, H., Xia, J., Liu, H., Wu, Y., Zhang, L., Yu, Z., Fang, M., Yu, T., Wang, Y., Pan, S., Zou, X., Yuan, S. and Shang, Y. (2020) Clinical course and outcomes of critically Ill patients with SARSCoV-2 pneumonia in Wuhan, China: a single-centered, retrospective, observational study. The Lancet Respiratory Medicine 8, 475-481.

Yin, Y. and Wunderink, R. G. (2018a) MERS, SARS and other coronaviruses as causes of pneumonia. Respirology 23, 130-137.

Zochios, V. and Jones, N. (2014) Acute right heart syndrome in the critically Ill patient. Heart Lung Vessel 6, 157-170.

Zochios, V., Parhar, K., Tunnicliffe, W., Roscoe, A. and Gao, F. (2017) The right ventricle in ARDS. Chest 152, 181-193. 